\title{
Fuzzy Improved Adaptive Delay Multicast Routing Protocol
}

\author{
B.Ravi Prasad \\ Research scholar, CSE Dept \\ GIT, GITAM University \\ RRSCET, Hyderabad
}

\author{
A.Damodaram \\ Professor in CSE \\ Director, AAC \\ JNTU, Hyderabad
}

\author{
G.Venkateswara Rao \\ Associate Professor, Dept of IT \\ GIT, GITAM University \\ Visakhapatnam
}

\begin{abstract}
A Mobile Ad-Hoc Network (MANET) represents a system of wireless mobile nodes that can freely and dynamically self organize into arbitrary and temporary network topologies without presence of any fixed infrastructure. Multicast routing in MANETs is an efficient method to lead data packets from one source group to several nodes as destination group. Multicasting can improve the efficiency of the wireless link when sending multiple copies of messages by exploiting the inherent broadcast property of wireless transmission. Although multicast routing algorithms in MANETs could be efficient in many situations, but the devices in MANETs are typically equipped with limited energy supplies which makes energy efficiency as one of the primary objectives in the design of multicast routing algorithms in wireless networks. In this paper fuzzy logic method is used to create small, strong forwarding group and reducing overhead method is used to restrict the domain of control packet flooding so as to reduce the overhead. The combination of above two methods is applied to Adaptive delay multicast routing protocol (ADRP). A ns-2 simulation study performed and our results revealed that the resultant increases packet delivery rate reduces average end-to-end delay and consumed power.
\end{abstract}

\section{General Terms}

Theory and Protocol

\section{Keywords}

Mobile Ad hoc networks, Multicast Routing, Adaptive Delay Multicast Routing, Fuzzy method, NS-2

\section{INTRODUCTION}

A mobile ad-hoc network (MANET) [1] is an autonomous collection of mobile nodes that communicates over bandwidth constrained wireless links. This network is not supported by any fixed infrastructure or central administration. The nodes are self organized and can be deployed anywhere, any time to support a particular purpose. Typically application areas of it includes battle fields, rescue sites and data acquisition in remote areas. An ad-hoc network is also useful in conventions and classrooms where participants share information dynamically.

In a typical ad hoc environment, network hosts work in groups to carry out a given task. Hence, multicast data transfer is more predominant than unicast data transfer. In military networks, multicast traffic dominates due to need of group communications. Multicasting involves the transmission of a datagram to a group of zero or more hosts identified by a single destination address, and is intended for group oriented computing. The use of multicasting within MANETs has many benefits. It can improve the efficiency of wireless channel while sending multiple copies of same data to different hosts. Instead of sending data via multiple unicast, multicasting minimizes channel consumption, sender and router processing, energy consumption and delivery delay.

Multicast routing [16] in MANETs is much more complex than in wired networks and faces several challenges. Multicast group members move, which prevents the use of a fixed infrastructure multicast topology. Various multicast protocols have been proposed to perform multicasting in ad-hoc networks. Multicast Routing protocols for MANETs have traditionally used shortest path routing to obtain paths to destinations, and do not consider traffic load or delay as an explicit factor. ADRP [4] gives a path source to destinations in which the delay is less than mean delay which is extension of wardrop routing in wireless networks [3]. The main concern is that overhead that ADRP create. ADRP uses control packets to establish the delivery structure which leads to high overhead. Minimizing such overhead is crucial for high performance as such control packets might also collide with data packets leading to increased packet loss. In this paper, fuzzy method and reducing overhead method are applied on ADRP to achieve low end to end delay, high packet delivery ratio and very low power consumption.

This paper is organized as follows: Section 2 provides survey related work and classification of multicast protocols. Section 3 provides description of ADRP. Section 4 describes fuzzy method and reducing overhead method. Section 5 describes simulation environment. Section 6 provides simulation results and concluding remarks in section 7 .

\section{RELATED WORK}

Multicasting plays a crucial role in many applications of mobile ad hoc networks. It can significantly improve the performance of these networks, the channel capacity (in mobile ad hoc networks, especially single-channel ones, capacity is a more appropriate term than bandwidth, capacity is measured in bits/s and bandwidth in $\mathrm{Hz}$ ) and battery power of which are limited. In the past couple of years, a number of multicast routing protocols have been proposed. In spite of being designed for the same networks, these protocols are based on different design principles and have different functional features when they are applied to the multicast problem. These protocols must deal with a number of issues, including, but not limited to, high dynamic topology, limited and variable capacity, limited energy resources, a high bit error rate, a multi hop topology, and the hidden terminal problem.

\subsection{Classification of Multicast Routing Protocols}

Multicast routing protocols can be classified based on construction of distributed paths in it, acquisition and maintenance of routing information, establishment of multicast connectivity and maintenance of multicast groups. 


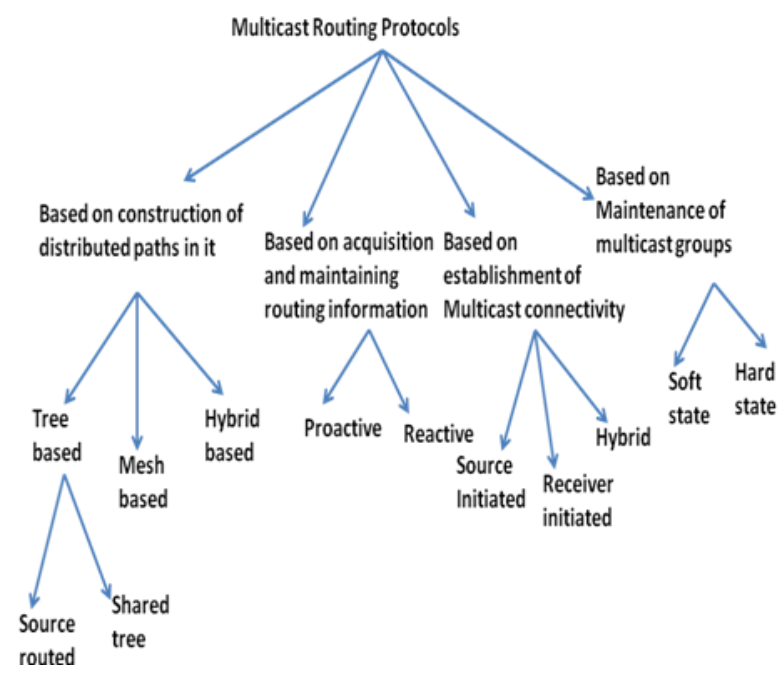

Figure 1: Classification Multicast Routing Protocols

\subsubsection{Classification Based on Construction of Distributed paths in it}

Multicast routing protocols for MANETs can be classified based on how distribution paths are constructed among group members. According to this, existing multicast protocols for MANETs can be divided into tree based(e.g.,MAODV[17], ABAM[13], ADMR[11]),AMRoute[8] mesh based(e.g.,ODMRP[9],CAMP[18][19][20][14],NSMP[12] and hybrid multicast protocols.

\subsubsection{Classification Based on Acquisition and Maintenance of Routing information}

Another classification method is based on how routing information is acquired and maintained by mobile nodes. Using this method, multicast routing protocols can be divided into proactive routing (e.g., CAMP [18] [19] [20] [14] and AMRIS [15]) and reactive routing (e.g., ACMRP [7] and ABAM [13]).

\subsubsection{Classification Based on Establishment of Multicast Connectivity}

Based on how multicast connectivity is established and maintained, multicast routing protocols are classified into the following two approaches. (i) The Source-Initiated approach (e.g., ODMRP [9]) (ii) The Receiver-Initiated approach (e.g., DDM [10]).

\subsubsection{Classification based on Maintenance of multicast groups}

MANETs suffer from frequent link breaks due to the lack of mobility of the nodes, which makes efficient group maintenance necessary. Maintaining the multicast group can be achieved by either the Soft-State approach or the HardState approach.

The main problem of many multicast routing protocols is their high data and control overhead which might also lead to low packet delivery probability due to collisions. Also, multicast delivery structures should be robust, e.g. composed of nodes which have high available capacity and good links in order to increase packet delivery further. The method which is proposed in this paper is not only reduces overhead significantly but also allows creating robust delivery structures using fuzzy based control in ADRP.

\section{ADAPTIVE DELAY MULTICAST PROTOCOL OVERVIEW}

ADRP [4] is mesh based source initiated multicast routing protocol which includes the neighboring concept and load adaptive concept. The routes are built and maintained using traditional request and reply messages. A soft state approach is used for multicast group maintenance.

\subsection{Different Steps in ADRP}

Step 1: Neighbor Awareness in ADRP

In ADRP each node keeps the information of all of its neighbours of one-hop distance in a neighbour table. Node periodically transmits HELLO packet shown in Figure 2 to find out its neighbour information.

\begin{tabular}{|l|l|l|l|l|}
\hline Type & $\begin{array}{l}\text { Source } \\
\text { ID }\end{array}$ & Sequence & $\begin{array}{l}\text { Neighbor } \\
\text { ID }\end{array}$ & $\begin{array}{l}\text { Neighbor } \\
\text { Delay }\end{array}$ \\
\hline
\end{tabular}

Figure 2: HELLO packet

\section{Step 2: Creation of Multicast Mesh}

In ADRP, new source initially sends a ROUTE-REQ packet shown in Figure.3. The ROUTE-REQ packet has a data payload field. When an intermediate node receives the ROUTE-REQ packet, it caches the upstream node and updates the field with its own address before forwarding it to next nodes. When a receiver receives the ROUTE-REQ packet, it sends a REP packet to the node from which it received the packet. The upstream node receives the REP packet and adds an entry for the group to its routing table. Then it forwards the REP packet to its own upstream node and the REP packet eventually reaches the source node. The intermediate nodes that relay the REP packet become forwarding nodes. The forwarding node information is maintained in Forwarding group table. A multicast mesh of a group consists of sources, receivers, forwarding nodes, and links connecting them. The nodes in a multicast mesh are called mesh nodes. After receiving the all the reply packets, at source node mean delay is calculated. Out of all the paths between sources to destination, the path which has lesser delay than mean delay is selected for data transmission. By considering all the possible paths between sources to multiple destinations, multicast mesh is created.

\begin{tabular}{|c|c|c|}
\hline Type & Sequence no & Reserved \\
\hline Source id & Neighbor id & Destination id \\
\hline FC & Delay & Pay load \\
\hline
\end{tabular}

Figure 3: ROUTE-REQ packet

\section{Step 3: Multicast Mesh Maintenance}

Each source node periodically transmits a LOCAL-REQ packet shown in Figure. 4 and only mesh nodes and group neighbor nodes relay the packet. Therefore, all nodes two hops away from the mesh nodes receive the LOCAL-REQ packet. This mechanism repairs most link failures caused by node movements. REP packets to LOCAL-REQ packets are relayed to a source in the same way as REP packets to ROUTE-REQ packets. Forwarding nodes and group neighbor nodes along a multicast mesh are updated as REP packets are relayed to a source. 


\begin{tabular}{|c|c|c|}
\hline Type & Sequence no & Reserved \\
\hline Source id & Mesh node id & Destination id \\
\hline FC & Delay & Pay load \\
\hline
\end{tabular}

Figure 4: LOCAL-REQ packet

\section{Step 4: DATA Packets Transmission}

When a node receives a DATA packet, it consults DataCache to see if the packet is duplicate. If so, it discards the packet. Otherwise, it updates DataCache to reflect the packet header information, especially the sequence number. And the packet is re-broadcast if the receiving node is a forwarding node.

ADRP has many advantages but it suffers from high overhead. This overhead is attributed mainly due to the mesh delivery structure and the network wide broadcasting of ROUTE-REQ packets. When there are many nodes or multicast sources in the network, data and control overhead increases significantly, especially for large networks.

Therefore, one important point to consider is how to reduce the overhead for the mesh creation and maintenance.

\section{FUZZY IMPROVED ADRP}

In this section, two main approaches are used for increasing the performance of multicast routing in a MANET. First, fuzzy logic based approach [5] is used to deal with imperfect knowledge about link and node characteristics. Second, the domain of control packet flooding [6] is restricted to reduce the overhead. Finally, how these approaches can be integrated into Adaptive delay Multicast Routing Protocol is demonstrated to reduce overhead.

\subsection{Fuzzy Logic Based Approach}

In the Mobile Ad-hoc Network, nodes are classified as strong and weak nodes. The strong node has properties like high power level, high bandwidth availability, low loss rate and low moving speed. A strong forwarding group is formed by using strong nodes. The probability of data delivery is increased by using strong forwarding group in the path. These strong forwarding groups are formed by using fuzzy logic based approach which should lead to decreased resources consumption and higher stability of the delivery structure.

In ADRP, any node which receives a ROUTE-REQ packet it catches the upstream node and updates the field with its own address before forwarding to next nodes. It does not consider whether the node is strong or weak from which it receives.

Several fields are added to the ROUTE-REQ packet shown in Figure 5.which carry extra information on e.g. bandwidth availability, loss rate experienced, moving speed, and power level to allow the nodes to perform a better route selection in the route request process. Based on such information, the next nodes will be able to compute the probability of caching and forwarding the received ROUTE-REQ message.

\begin{tabular}{|c|c|c|c|}
\hline Type & Sequence & Source ID & Neighbor ID \\
\hline Bandwidth & Loss & Speed & Power \\
\hline Query & $\begin{array}{c}\text { Destination } \\
\text { ID }\end{array}$ & FC & Hop count \\
\hline Delay & \multicolumn{2}{|c|}{ Number of previous forwarding group } \\
\hline
\end{tabular}

Figure 5: Modified ROUTE-REQ packet
Once a node receives a ROUTE-REQ packet, it needs to process the parameters like bandwidth, speed, power and loss rate of the previous node. To process the above parameters, node needs to use fuzzy logic to handle network dynamics, imprecise information and uncertainty. A simple membership function is used to fuzzify parameters. The value of node parameters are shown in horizontal axis and membership probability is shown in vertical axis. By using the parameters value node have to classify them as low, medium and high probability nodes. Before forwarding node replaces its own parameters information in ROUTE-REQ packet.

Figure 6 show each node's decision process based on the fuzzy logic. Input to this process is the previous node's operating parameters (such as bandwidth, speed, power and loss rate ) where the probability of catching and forwarding is the output of the process.

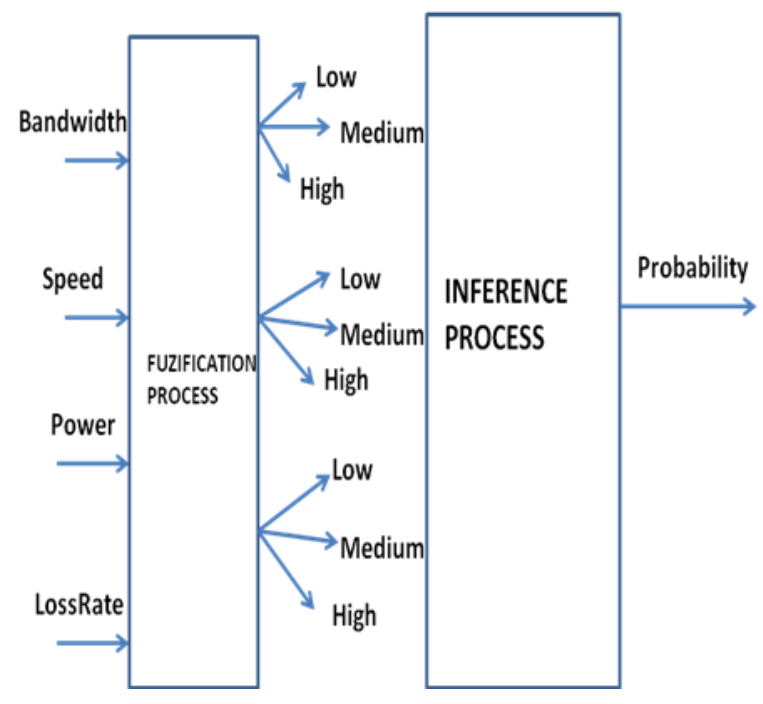

Figure 6: Fuzzification Process

In the inference stage of the fuzzy process, inference laws shown in Algorithm 1 are used to compute the probability of caching and forwarding based on the simple rules.

If ((bandwidth is high) and (power is high) and (speed is low)and(loss rate is low)) then Increase prob. of forwarding ROUTE-REQ packet

If ((bandwidth is low) and (power is low) and (speed is high)and(loss rate is high)) then Decrease prob. of forwarding ROUTE-REQ packet

If ((bandwidth is low) and (power is high) and (speed is medium)and(loss rate is medium)) then Do not change prob. of forwarding ROUTE-REQ packet

\section{Algorithm 1: Inference Rules}

\subsection{Reducing the Overhead}

In ADRP, Hello packets are periodically send to find out neighbors, ROUTE-REQ packets are used to construct mesh and LOCAL-REQ packets are used to maintain session. All these packets are lead to increase overhead in the mobile ad hoc networks. In the reducing the overhead method, the new forwarding group can be established from the current forwarding group which leads to decrease in the mobility of ROUTE-REQ packets. To implement this idea, Number of previous forwarding group field is added to ROUTE-REQ packet. If a ROUTE-REQ packet has visited many nodes but 
it does not see any previous forwarding group nodes, then the packet will be discarded. Therefore, when a node receives a new ROUTE-REQ packet, it extracts NOPFG (Number of previous forwarding group) and Hop count fields from the incoming packet. Hop count field is the number of hops to this node from the sender. When a node receives a ROUTE-REQ packet with a hop count greater than the minimum value, it decides if the ROUTE-REQ packet will be forwarded or discarded based on a random value. This random value is based on the forwarding probability which is calculated by fuzzy model (Figure 6). The minimum value of hop count allows a ROUTE-REQ packet to traverse sufficient number of hops to prevent from discarding all copies of ROUTE-REQ packets.

This method does not work for very low density networks. So, before using this, network is to be checked. After checking by using algorithm 2, it is decided to use Fuzzy improved ADRP or simple ADRP to construct mesh in the network.

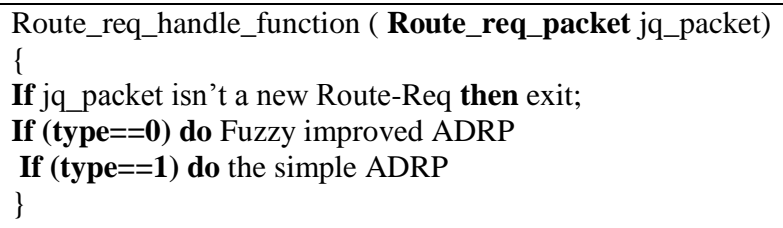

\section{Algorithm 2: ROUTE-REQ Handle}

\subsection{Fuzzy Improved ADRP Method}

The combination of fuzzy logic based approach and reducing the overhead method is applied on ADRP is to reduce the overhead. The following algorithm 3 shows Fuzzy improved ADRP method function.

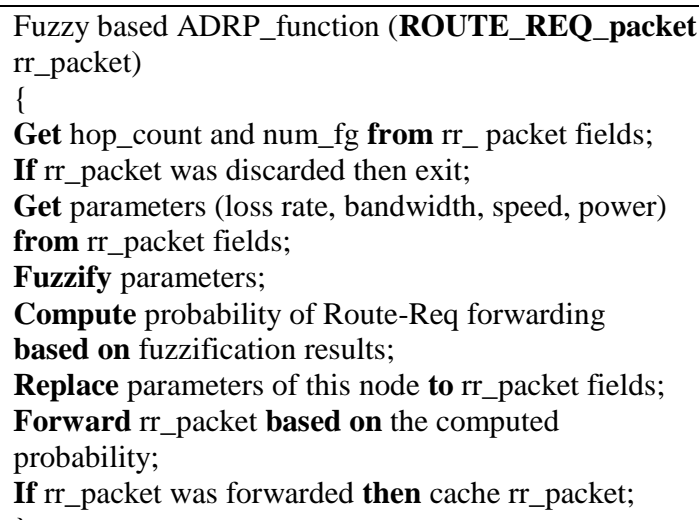

\section{Algorithm 3: Fuzzy Improved ADRP}

In the above algorithm rr_packet is discarded when it has travelled several hops but not seen any node from previous forwarding group.

\section{SIMULATION ENVIRONMENT}

$N S-2$ simulator was used for performance simulation. NS-2 is originally developed by the University of California at Berkeley and the VINT project and extended to provide simulation support for ad hoc networks by the MONARCH project [21] at Carnegie Mellon University. Reference [22] gives a detailed description about physical layer, data link layer, and IEEE 802.11 MAC protocol used in the simulation. Recently VINT project [2] gives extensions to ns-2 simulator.
Our simulation modeled a network of up to 500 mobile nodes that were placed randomly within $1000 \mathrm{~m} \times 1000 \mathrm{~m}$ area. Radio propagation range for each node was 250 meters and channel capacity was $6 \mathrm{Mbits} / \mathrm{sec}$. Nodes move according to the "random way-point" model which is characterized by a pause time. A pause time of 10 seconds was used in the simulation. Each movement scenario was made on the basis of the model. Member nodes were randomly selected. Each member node joins at the beginning of the simulation and remains as a member throughout the simulation. Each multicast source sends two 512-byte packets per second and averaged 10 runs with different movement scenarios and each simulation executed for 80 seconds of simulation time.

Table 1: Simulation Environment

\begin{tabular}{|l|l|}
\hline Area & $1000 \mathrm{~m} * 1000 \mathrm{~m}$ \\
\hline Radio Propagation range & $250 \mathrm{~m}$ \\
\hline Channel capacity & $6 \mathrm{Mbits} / \mathrm{sec}$ \\
\hline Pause time & $10 \mathrm{sec}$ \\
\hline Simulation time & $80 \mathrm{sec}$ \\
\hline Packet size & 512 bytes \\
\hline
\end{tabular}

The default values for the fuzzy membership functions are the following: low and medium values for speed are $6 \mathrm{~m} / \mathrm{s}$ and 12 $\mathrm{m} / \mathrm{s}$; low and medium values for power are 0.3 and 0.5 ; low and medium values for bandwidth are 2 mbps and 4 mbps. In these memberships if input value is greater than medium value it's a high value.

One member node sends constant bit rate traffic with 5 packets per second to all other member nodes from start to end of the simulation. Fuzzy Improved ADRP has been simulated and compared with ADRP.

\section{SIMULATION RESULTS}

To evaluate the performance of the proposed methods, the following parameters have been analyzed:

- End to end delay: The average end-to-end delay from a transmission of the packet to a successful reception at a receiver.

- Number of received packets: Total number of packets which have been received during the simulation period. This allows calculating packet loss rate.

- Overall overhead: Total traffic in network.

\subsection{Impact of number of nodes}

If the number of nodes increases in the network then in ADRP overhead increases because frequent transmission of HELLO, ROUTE-REQ, LOCAL-REQ packets. But in the case of Fuzzy improved ADRP transmission of ROUTE-REQ and LOCAL-REQ, packets is less, so overhead is less in the case of Fuzzy improved ADRP. This section analyzes performance of Fuzzy improved ADRP under increasing of number of nodes. 


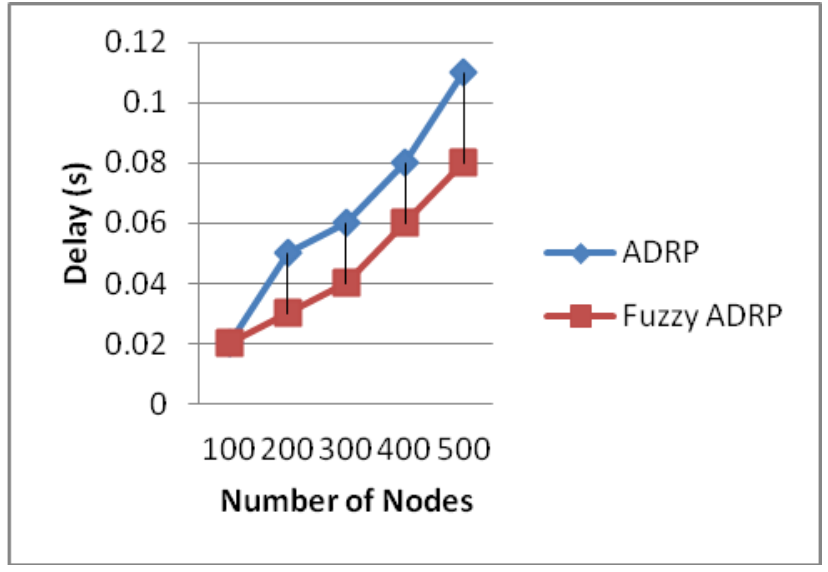

Figure 7 : Delay vs Number of Nodes

Figure 7 compares end to end delay of Fuzzy improved ADRP and ADRP under increase of number nodes. If number of nodes increases in network then in ADRP the participating nodes in ROUTE-REQ packet forwarding and LOCAL-REQ packet forwarding also increases which leads to loss rate and delay increases. But in fuzzy ADRP the increment of loss rate and delay is less when compared it to ordinary ADRP because fuzzy method uses strong forwarding group and new forwarding group is established from the current forwarding group.

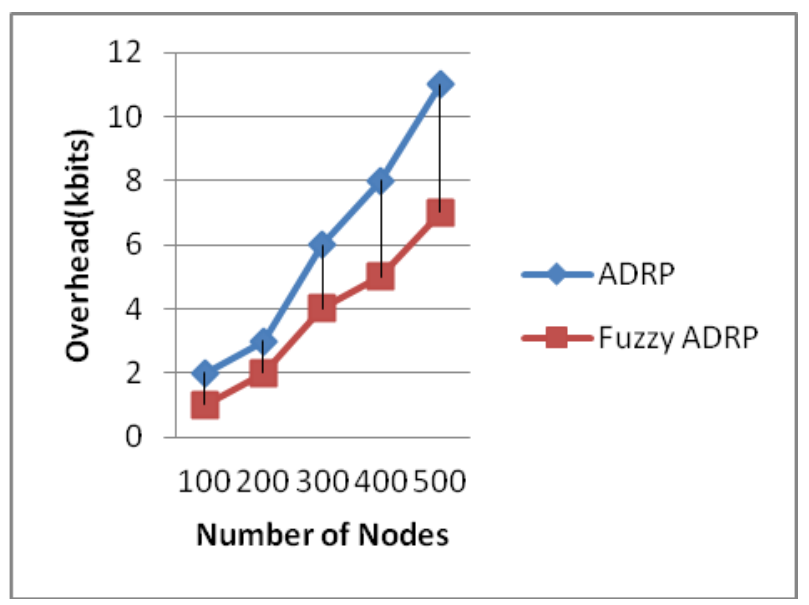

Figure 8: Overhead vs Number of Nodes

Figure 8 compares overhead of Fuzzy improved ADRP and ADRP under increase of number of nodes. When the number of nodes increases in the network, overhead increases more in ADRP compared to Fuzzy improved ADRP.

Standard ADRP delivers higher overhead to network resulting in higher delay and higher probability for collisions. Fuzzy ADRP gives the best performance due to the limitation of the flooding domain.

A higher overhead leads to higher power consumption and lower network life time. Again, fuzzy has lowest power consumption due to the lowest overhead.

\subsection{Impact of Speed}

Multicast routing protocols faces many challenges with nodes mobility. Nodes move in network with different speed. This section analyzes the performance of Fuzzy improved ADRP under increases of node speed.

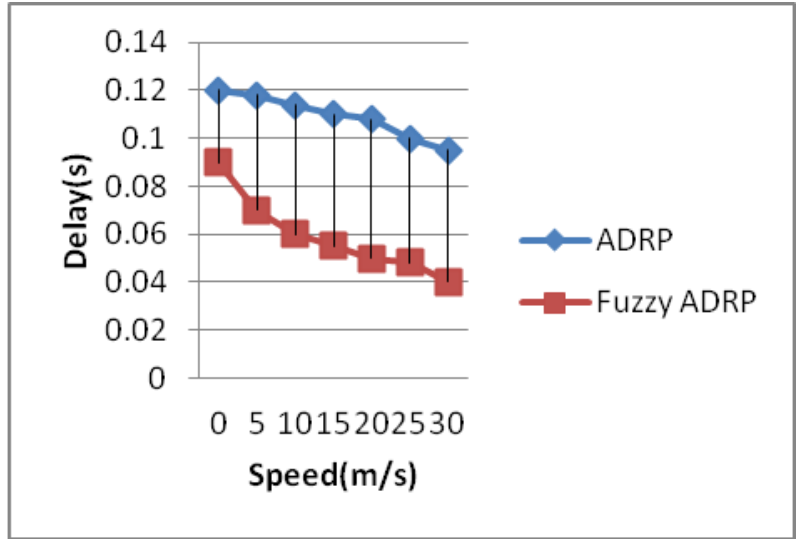

Figure 9: Delay Vs Speed

Figure 9 shows the Fuzzy improved ADRP has less delay compared standard ADRP. In Fuzzy improved ADRP, the new forwarding group can be established from the current forwarding group which leads to decrease in the mobility of ROUTE-REQ packets. Even though speed of nodes increases because of less mobility of ROUTE-REQ packets delay decreases in case of Fuzzy improved ADRP. But, in standard ADRP delay is higher than Fuzzy improved ADRP because of more mobility of ROUTE-REQ packets.

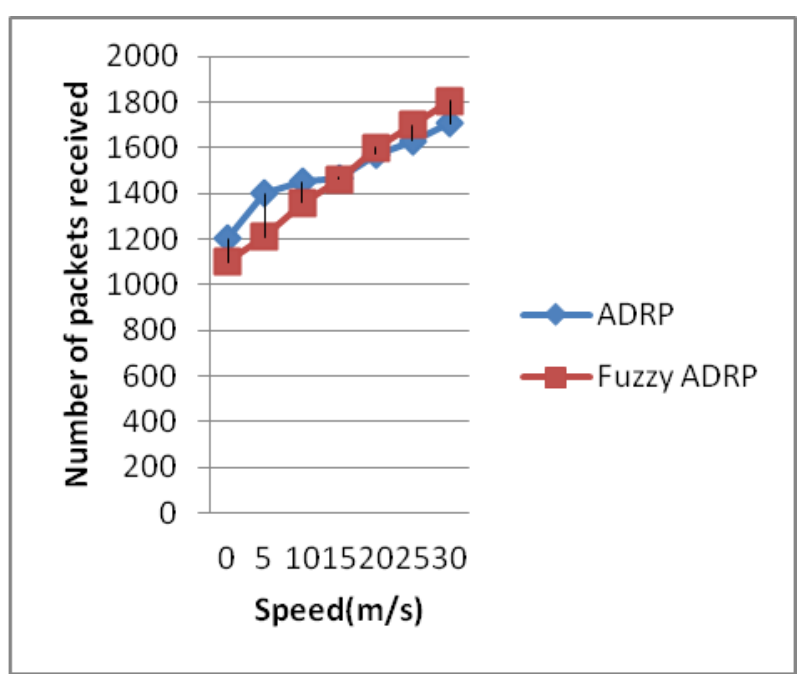

Figure 10: Number of Packets Received Vs Speed

Figure 10 compares number of packets received in Fuzzy improved ADRP and ADRP under increase of speed. Fuzzy improved ADRP uses strong forwarding group to transfer data from source to destinations, so breakage of links in Fuzzy improved ADRP is less when compared to ADRP. So Number of packets received in Fuzzy improved ADRP is more when node speed is more.

In case of high mobility ADRP suffers from high overhead. This high overhead leads to more power consumption. It leads to frequent node failures, leads to less number of receiving nodes when compared to Fuzzy based ADRP.

\subsection{Impact of Traffic Density}

This section analyzes the performance of Fuzzy improved ADRP and standard ADRP under increase of traffic density. 


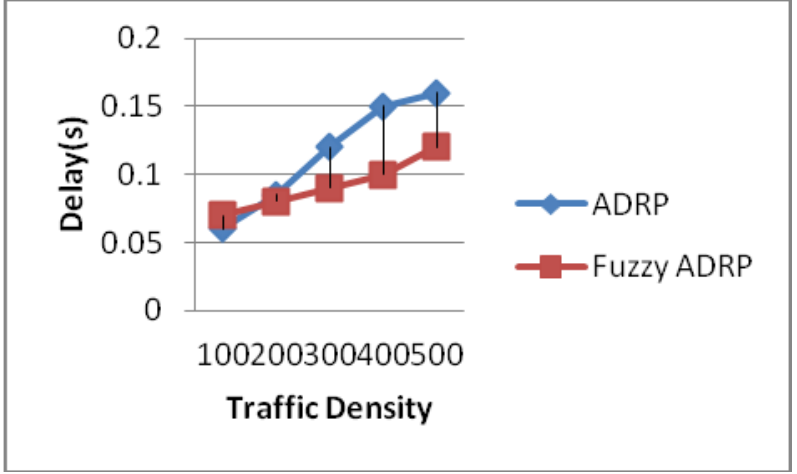

Figure 11: Delay vs. Traffic density

Figure 11 shows end to end delay for all simulated methods. The standard ADRP floods data packets in a weak forwarding group. The proposed Fuzzy improved ADRP try to form a small and good forwarding group. A small forwarding group has very low overhead in comparison to standard ADRP. Therefore the proposed method has very low end to end delay in comparison to standard ADRP.

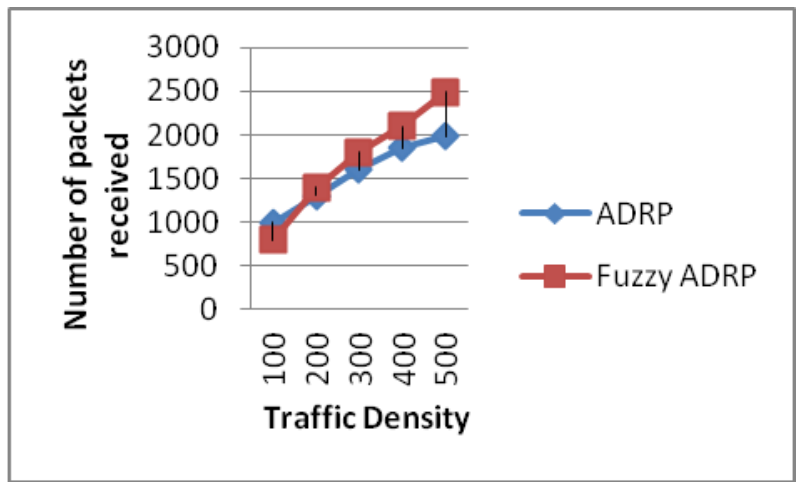

Figure 12: Number of packets received vs Traffic density

Figure 12 present number of received data packet by two methods. The fuzzy method use good nodes and routes to forward data packet. Therefore they deliver very high number of data packets. Also it forms a small and strong forwarding group instead great and weak forwarding group.

\section{CONCLUSION}

In this paper, Fuzzy based approach is used to improve performance of multicast routing algorithms in MANETs specially the ADRP. Firstly fuzzy logic tries to construct a small and efficient forwarding group instead of great and inefficient one. Secondly reducing overhead method tries to reduce the domain of control packet flooding, it achieve more improvement in the reduction of control overhead. These two methods collectively applied on ADRP and developed Fuzzy improved ADRP. Regard to simulation results, Fuzzy improved ADRP model has many advantages to the standard ADRP such as lower control and data overhead, low end to end delay, high packet delivery ratio and very low power consumption. Thus it can be used in many applications in ad hoc networks.

In the future work, Reducing the overhead method can be applied to low dense networks.

\section{REFERENCES}

[1] Internet Engineering Task Force (IETF) Mobile AdHoc Networks (MANET) Working Group
Charter.http://www.ietf.org/html.charters/manetcharter.html.

[2] Kevin Fall, and Kannan Varadhan, editors, "ns notes and documentation," The VINT Project, UC Berkeley, LBL, USC/ISI and Xerox PARC, Nov.2011. Available at http://www.isi.edu/nsnam/ns/ns-documentation

[3] Raghunathan $\mathrm{v}$ and Kumar P.R. "wardrop routing in wireless networks" in IEEE transactions on mobile computing, May 2009 Issue 5 pages 636-652

[4] B Ravi Prasad, Dr.A.Damodaram, Dr.G.Venkateswara rao "Implementation of Adaptive Delay Multicast Routing Protocol" in IJARCCE March 2013

[5] Shams Shafigh, A., K. Abdollahi and A.J. Kassler,2010. Improving performance of ODMRP by Fuzzy Logic Control.WCNIS2010, China.

[6] Abdollahi, K., A. Shams Shafigh and A.J. Kassler, 2010. Improving performance of ODMRP by Deleting Lost Join Query Packets. ACIT2010, Spain.

[7] S. Park and D. Park, "Adaptive core multicast routing protocol," Wireless Networks, vol. 10, no. 1, pp. 53-60, 2004.

[8] J. Xie, R. R. Talpade, A. McAuley, and M. Liu, "AMRoute: ad hoc multicast routing protocol," Mobile Networks and Applications, vol. 7, no. 6, pp. 429-439, 2002.

[9] S. J. Lee, W. Su, and M. Gerla, "On-demand multicast routing protocol in multicast wireless mobile networks," ACM/Baltzer Mobile Networks and Applications, Vol. 7, 2002, pp. 441-453

[10] L. Ji and M. S. Corson, "Differential destination multicast-a MANET multicast routing protocol for small groups," in Proceedings of the 20th Annual Joint Conference of the IEEE Computer and Communications Societies (INFOCOM '01), vol. 2, pp. 1192-1201, 2001.

[11] J. G. Jetcheva and D. B. Johnson, "Adaptive demanddriven multicast routing in multi-hop wireless ad hoc networks," in Proceedings of the 2nd ACM International Symposium on Mobile Ad Hoc Networking and Computing (MobiHoc '01), pp. 33-44, 2001.

[12] S. Lee and C. Kim, "Neighbour supporting ad hoc multicast routing protocol," in Proceedings of the ACM International Symposium on Mobile Ad Hoc Networking and Computing (MobiHoc 'O0), pp. 37-44, 2000.

[13] C-K. Toh, G. Guichal, and S. Bunchua. "ABAM: OnDemand Associativity-Based Multicast Routing for Ad Hoc Mobile Networks“, IEEE Vehicular Technology Conference 2000. 52nd Volume: 3, pp. 987-993, 2000.

[14] E. L. Madruga and J. J. Garcia-Luna-Aceves, "Scalable multicasting: the core-asisted mesh protocol," ACM/Baltzer Mobile Networks and Applications, Vol. 6, 2000, pp.151-165

[15] C. W. Wu, Y. C. Tay, and C.-K. Toh, "Ad hoc Multicast Routing protocol utilizing Increasing id-numberS (AMRIS)," draft-ietf-manet-amris-spec-00.txt, 2000.

[16] S. Lee, W. Su, and M. Gerla, "Ad hoc wireless multicast with mobilityprediction," IEEE ICCCN '99, Boston, MA, Oct. 1999. 
[17] E. M. Royer, C. E. Perkins. "Multicast Operation of the Ad-hoc On-Demand Distance Vector Routing Protocol", Proceeding of the fifth annual ACM/IEEE international conference on mobile computing and networking, pp. $207-218,1999$.

[18] J.J. Garcia-Luna-Aceves and E.L. Madruga, .The CoreAssisted MeshProtocol,. IEEE Journal on Selected Areas in Communications, vol. 17,no. 8, Aug. 1999, pp. 13801394.

[19] J.J. Garcia-Luna-Aceves and E.L. Madruga, .A Multicast Routing Protocolfor Ad-Hoc Networks,. In Proceedings of IEEE INFOCOM'99, NewYork, NY, Mar. 1999, pp. 784-792.
[20] E.L. Madruga and J.J. Garcia-Luna-Aceves, .Multicasting Along Meshesin Ad-Hoc Networks,. In Proceedings of IEEE ICC'99, Vancouver,Canada, Jun. 1999, pp. 314-318.

[21] "The CMU Monarch Project"s wireless and mobility extensions to ns," The CMU Monarch Project, Aug. 1999. Available at http://www.monarch.cs.cmu.edu/.

[22] J. Broch, D. A. Maltz, D. B. Johnson, Y. Hu, and J. Jetcheva, "A performance comparison of multi-hop wireless ad hoc network routing protocols," Proceedings of the Fourth Annual ACM/IEEE InternationalConference on Mobile Computing and Networking, ACM, Dallas, TX, Oct. 1998. 